\title{
中国种学
}

\section{远心区空气簇射粒子到达时间 分布的模拟计算}

\author{
戴 宏 跃 \\ （中国科学院离能物理研究所, 北京） \\ 笠原克昌 \\ (日本东京大学宇宙线研究所)
}

摘 要

本市通过 Mont Calro 模拟, 计算了远心区 $(r>100 \mathrm{~m})$ 宇宙线空气筷射粒子 的到达时间(延迟时间)分布。

时间分有是通过三种参数来表述的，我们导出了一种表达这种时间分有的近似 公式。计算发现这种时间分布对筇心距离有很强的依赖关系,但是,基本上独立于篍 射年龄、到达方向、原初能量以及原初成分。如果粒子数很少，事例与事例之间的到 达时间分布会有很明显的差别，这仅仅是由统计涨落所致。因此，如果要将模拟计算 与实验结果相比较，必须考虑实验的实际情况.

\section{关键词：宇宙线，广延大气簇射，到达时间分布，Mont Calro 模拟}

模拟所用的作用模型，详见文献[1]，在模拟程序(主要由笠原克昌发展)所给的几个作用 模型中,我们选用了现时认为最好的 QCD 模型.

级联过程的处理，在这样的一个到达付间分布的模拟计算中是至关重要的，在此，我们仅 仅说明三点:

1. 多重散射是用角度和活河分行相吴积的 Fermi 的 Gauss 近似来处理的，与 Moliere 的 散射理论没有朋显的区歫。

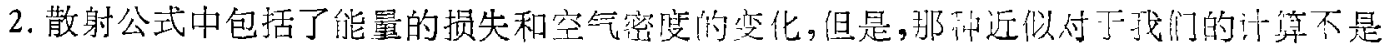
很合适，因此，在一次取样中电子及其他带电粒子沂涽通过的最长洛径受到限制，因而，粒子的

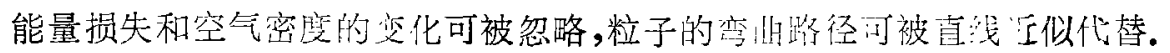

3. 由以上的近似䜣得到的煜子的路径比实际路桯约小 $10 \%$, 因此, 时间分行的䆓度大约 也小 $10 \%$ 。

\section{一、模拟结果}

在四维的空间里跟踪每一个粒子是很费机时的, 而且这种㸷费正比于原初能量, 因此, 我 


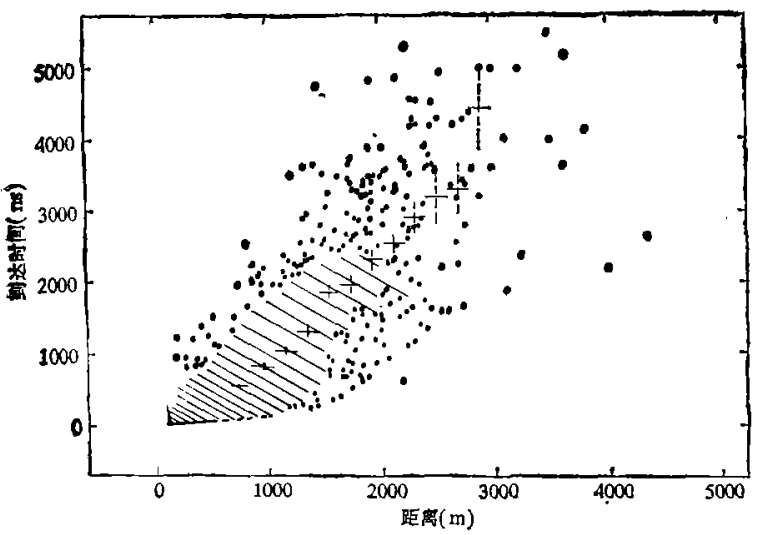

（a）空气射精子的到达时间分有的散点图(隄影部分 表示煜子的密慗区域,时间轴为线性坐标)

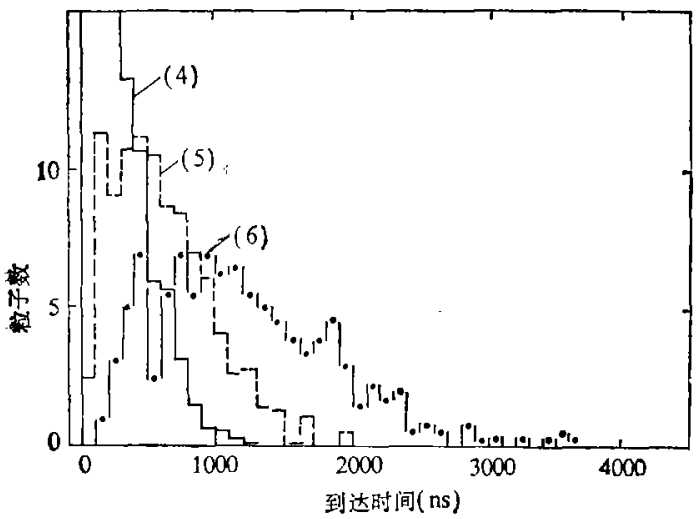

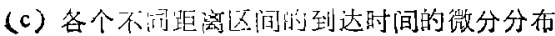
(区间的定义同图 1(d))

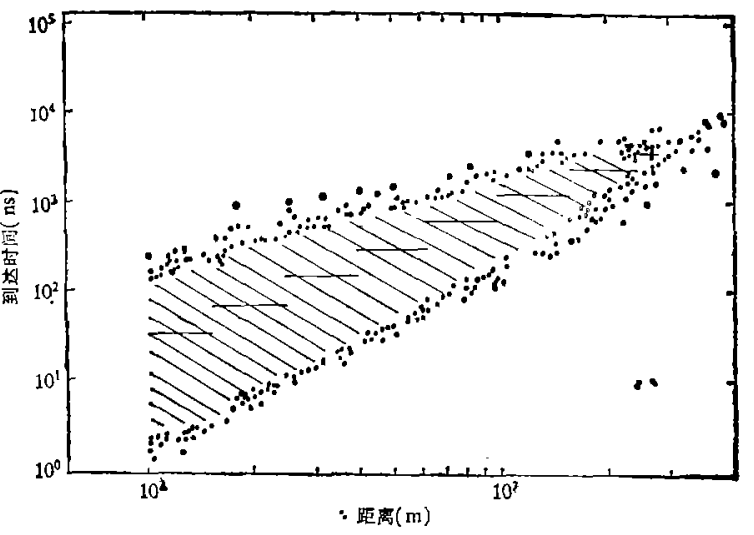

（b）空气簇射粒子的到达时间分布的散点图(阴影部分 表示粒子的密集区域,时间轴为对数坐标)

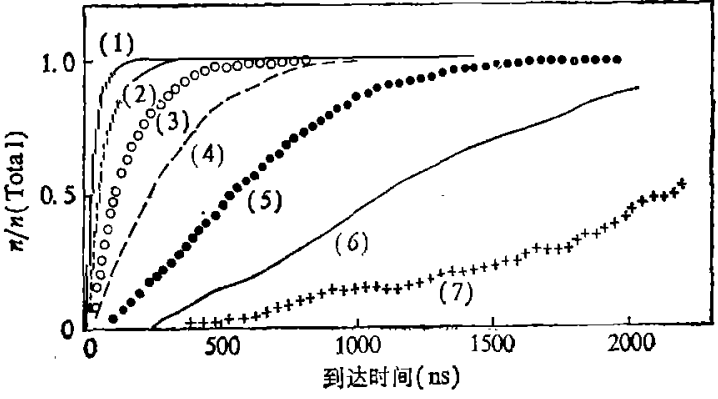

（d）不同距离区间的到达时间的积分分布 （图中的数字所代表的距离区间为:

(1) $-100 \mathrm{~m}<r<158 \mathrm{~m}$, (2) - $158 \mathrm{~m}<r<251 \mathrm{~m}$, (3)$251 \mathrm{~m}<r<398 \mathrm{~m}$, (4)- $398 \mathrm{~m}<r<631 \mathrm{~m},(5)-631 \mathrm{~m}<$ $r<1000 \mathrm{~m},(6)-1000 \mathrm{~m}<r<1580 \mathrm{~m},(7)-1580 \mathrm{~m}<r<$ $2000 \mathrm{~m}$ )

图 1 簇射粒子的到达时间分布

们模拟计算的能是设置在 $100 \mathrm{TeV}$ ，为了得到足够多的粒子，观测的高度设置在 $600 \mathrm{~g} / \mathrm{cm}^{2}$ (接 近 $500 \mathrm{~g} / \mathrm{cm}^{2}$ 的最大发展处). 根据以前的模拟经验而且在后面也将看到: 这种时间分布几可 是与原初能量、成分、观测高度无关的, 但是重要的是获得尽可能多的电子数 $N_{\mathrm{e}}$ 以便减小涨 落,这是我们选择 $100 \mathrm{TeV}$ 和 $600 \mathrm{~g} / \mathrm{cm}^{2}$ 的原因.

图 1 所示的是原初质子 $\sec \theta=1.0$ 的模找结果. 图 1(a) 是线性坐标下到达时间分斥的 散点图; 图 1(b) 是对数坐标下的散点图. 图 1(a) 和 (b) 中的十字和短线代表每个距离区间 的平均到达时间. 从图 1(a) 中我们发现在近心区到达时间的离散很小, 但这种离散随簇心距 离的塯大而迅速增加. 图 1(c) 所示的是几个不同距离区间的到达时间的微分分布; 图 1(d) 是积分分布. 从图 1(c) 中很容易发现, 直到簇心距离 $1500 \mathrm{~m}$ 处, 粒子主要集中在簇射波面的 
前沿部分, 而且伴随着一个很长的衰减尾巴. 如果距簇心太远, 由于粒子数很少，这种分布的! 特征不明显.

本文试用了三个参数来描述簇射粒子的到达时间分布：第一个参数是到达时间弥散 $\sigma$. 其定义为

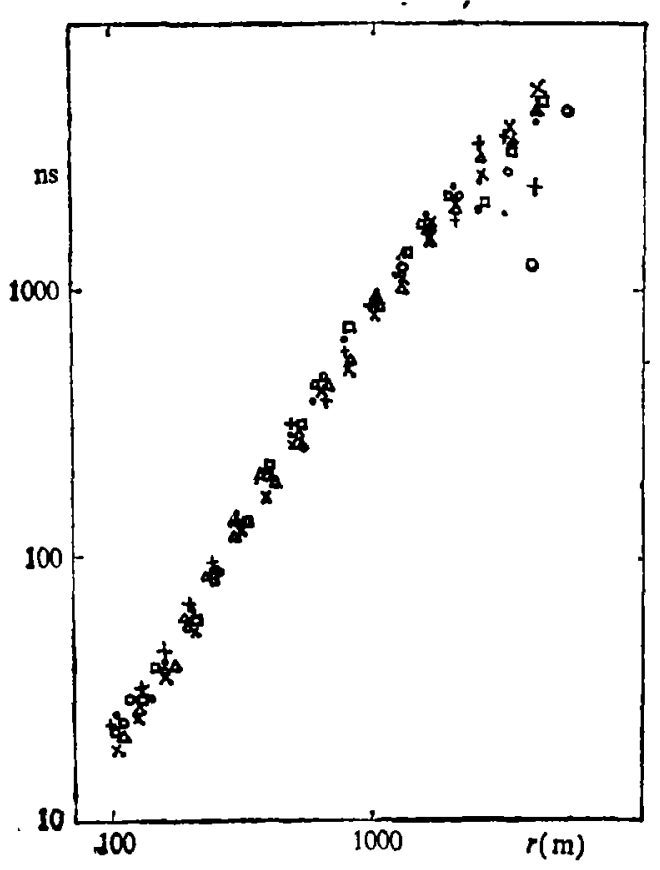

(a) $\tau, 100 \mathrm{TeV}, P$

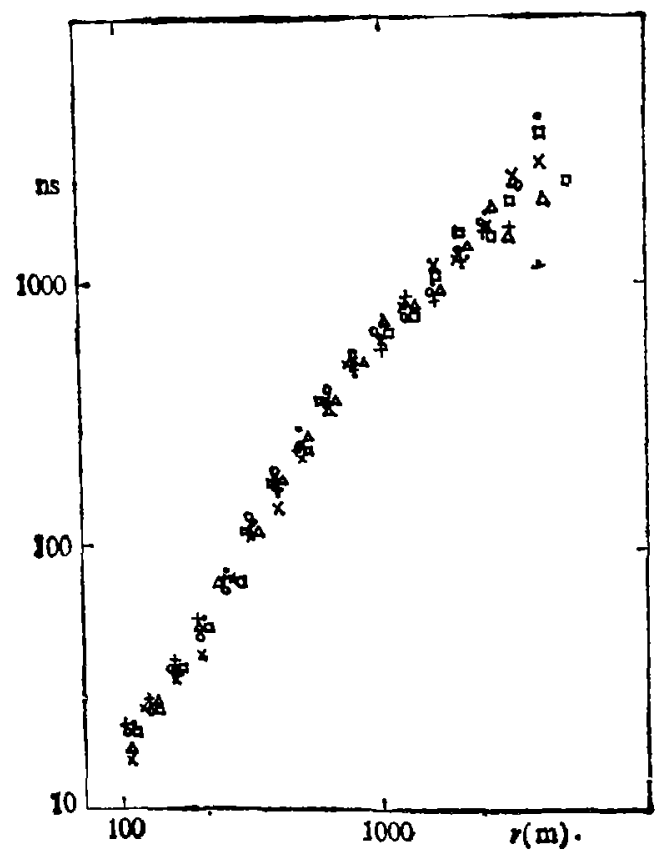

(c) $\iota_{r}, 100 \mathrm{TeV}, p$

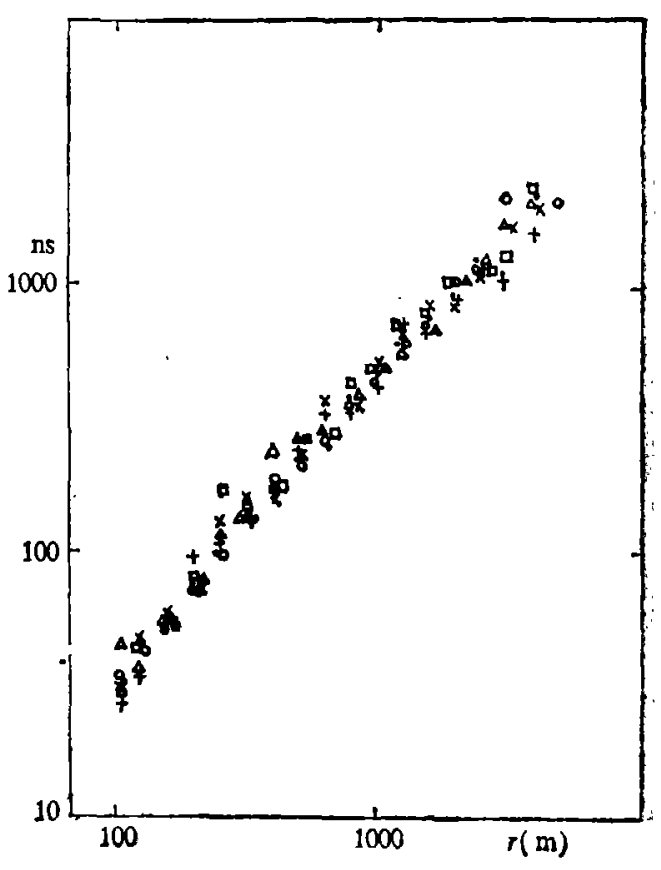

(b) $\sigma, 100 \mathrm{~T} \in \mathrm{V}, P$

图 2 不同天顶角 $\theta$ 下 $\tau, \sigma, s_{\mathrm{r}}$ 随，的变化. （原初质子，100个 TeV;

$\begin{array}{ll}\square-\sec \theta=1.0, & 0-\sec \theta=1.1, \\ +-\sec \theta=1.2, & x-\sec \theta=1.3, \\ \Delta-\sec \theta=1.4, & \square-\sec \theta=1.5)\end{array}$ 


$$
\sigma^{2}=\sum_{i=1}^{n}\left(t_{i}-\bar{t}\right)^{2} /(n-1),
$$

'其中 $t_{i}$ 为第 $i$ 个粒子的到达时间, $\bar{i}$ 为平均到达时间.

第二个参数用 $t$ ，表示，其定义为 $20-70 \%$ 积分时间分布之间的时间间隔.

正如从图 1(d) 中所看到的, 如果定义最先到达粓子的到达时间为 $t=0$, 那么, 到达时间 的积分分布很象一个 $e$ 指数型的分布(严格地说, 在远心区不成立). 因此, 我们用如下的一个 含有 $e$ 指数的公式来拟合到达时间的分布:

$$
F(t)=1-\exp (-t / \tau),
$$

其中 $\tau$ 即定义为第三个参数.

为了检验这种到达时间分布与人射方向 (天顶角 $\theta$ ) 之间的关系，我们进行了不同入射方 向的事例的模拟 $(\sec \theta=1.0,1.1,1.2,1.3,1.4,1.5$, 其中, $\sec \theta=1.0$ 为垂直人射). 图 2 是三种参数在不同 $\theta$ 下随距离的变化关系. 从图 2 中看不出任何明显的对 $\theta$ 的依赖关系 $(\theta$ 在 $0^{\circ}$ 至 $50^{\circ}$ 之间). 这意味着这种到达时间的分布是与第一次作用点的高度无关的. 比较图 2 的 (a)，(b) 和 (c), $\tau$ 和 $t_{r}$ 与簇心距离 $r$ 之间的关系明显地陡于 $\sigma$ 与 $r$ 的关系.

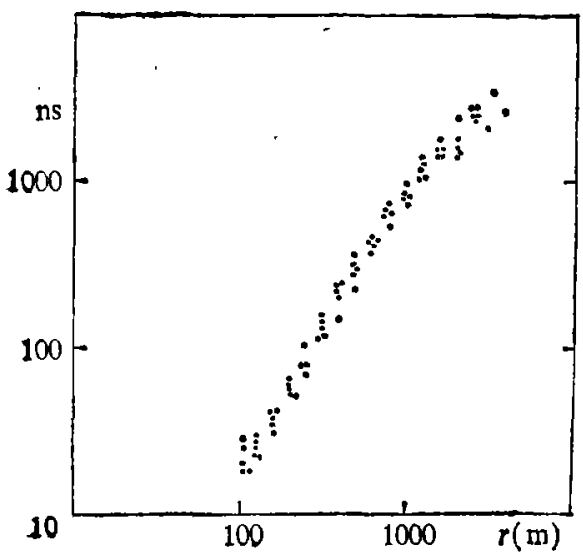

(a) $\tau, P, 50 \mathrm{TeV}$

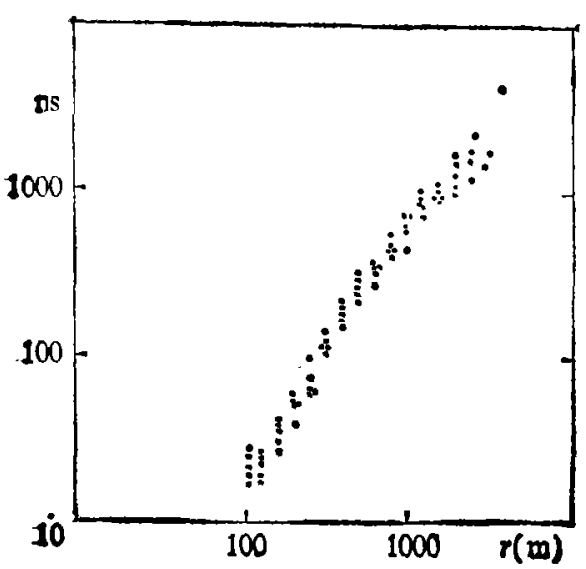

(c) $i_{r}, P, 50 \mathrm{TeV}$

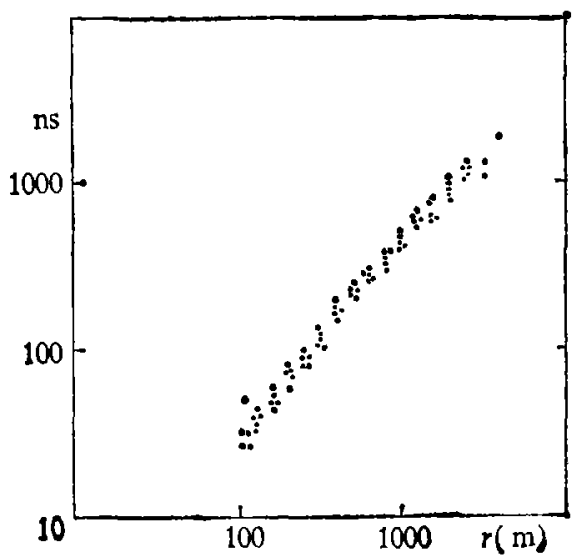

(b) $\sigma, P, 50 \mathrm{TeV}$

图 35 个不同事例的 $\tau, \sigma, t$ 的分布

(质子, $50 \mathrm{TeV} ; \sec \theta=1.0,5$ lvents) 
到达时间的涨落在远心区很大，这些距离区间的粒子数大约为 10-20，这意味着如果粒 子数不是足够的多, 这些参数都要遭受很严重的涨落, 这种因素限制了 Linsley Effect ${ }^{[2,3]}$ 的 应用。

为了验证事例与事例之间的到达时间的涨落，我们模拟了5个 $50 \mathrm{TeV}$ 的事例(原初质子， 垂直人射)，模拟结果见图 3. 除了最远的距离区间，事例与事例之间的胀落并不是很大，而在 最远的区间, 粒子数是有限的. 比较图 3 和图 2, 我们发现这三种参数对能量没有明显的依赖: 关系。

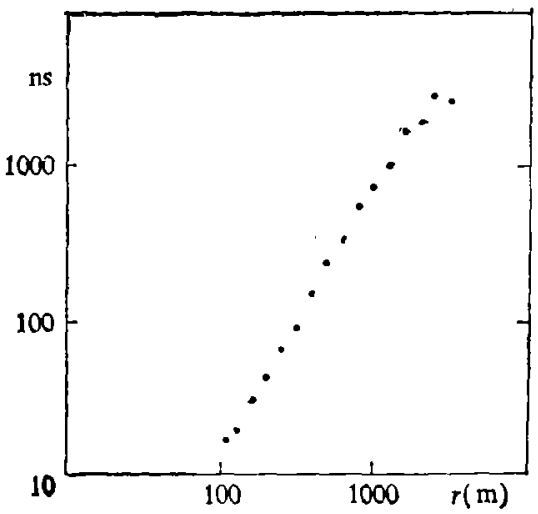

(a) $\tau$

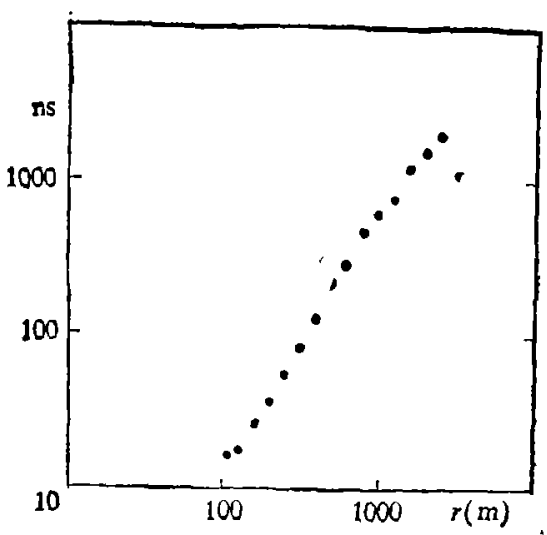

(c) $t_{r}$

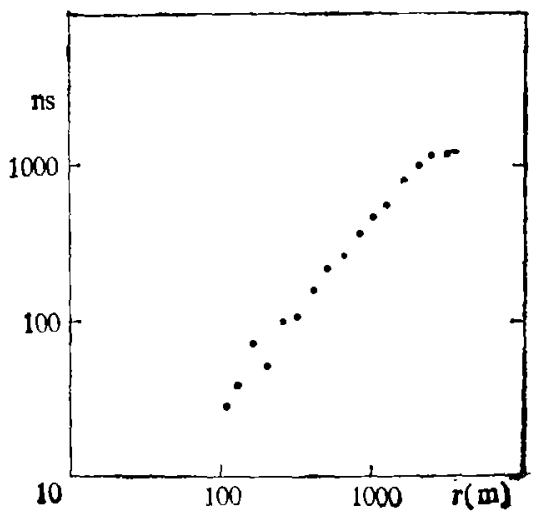

(b) 0
图 4 原初铁核的 $\tau, \sigma, t r$ 的分布 $(\mathrm{Fe}, 100 \mathrm{TeV}, \sec \theta=1.0)$

图 4 所示的是原初铁核所引起的簇射的模拟结果,与图 2 相比较,亦无明显的差别, 因此 可以推论到达时间的分布也是与原初成分无关的.

极少有强子被散射到距簇心 $100 \mathrm{~m}$ 以外处, 几乎所有的粒子都是电子（９0\%）和 $\boldsymbol{\mu}$ 子 $(\sim 10 \%)$. 在空气簇射的扁盘上, 最快的部分是高能 $\mu$ 子, 它们经过很少的散射而从很高的作 用点到达观测面. 而电子却要经过多重散射, 因此, 它们的到达时间(在对数坐标中)近似地表 现为 Gauss 分布 (对 Gauss 分布的偏移随距离的增加而增加). 次级粒子的种类以及能量的 分布见图 5 和图 6 . 


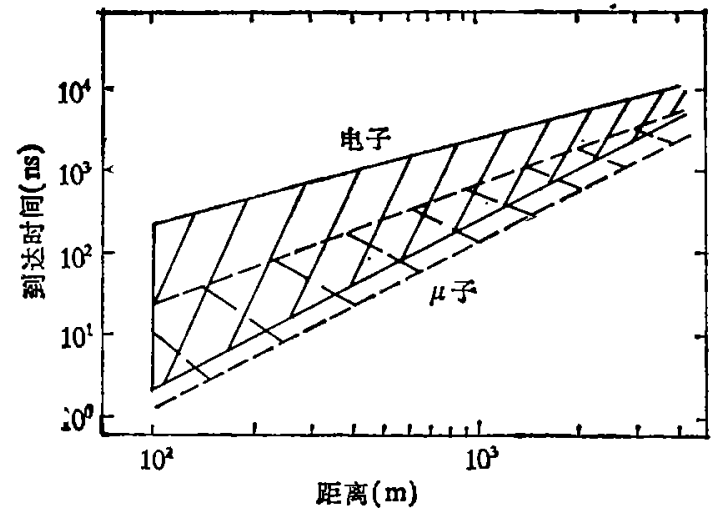

图 5 空气簇射中电子和 $\mu$ 子分布的区域

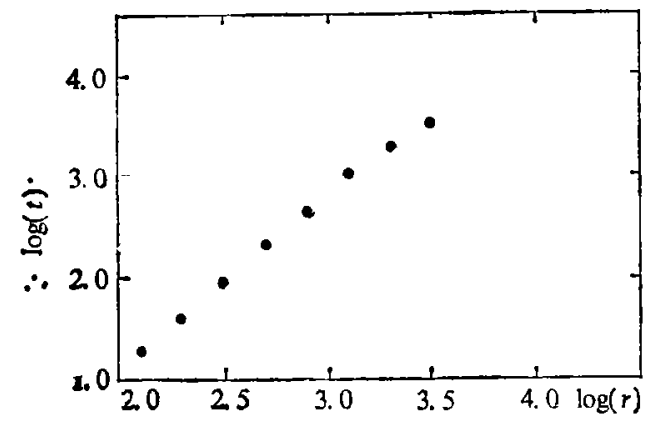

图 7 平均到达时间分布(对数坐标下) 随距离的关系

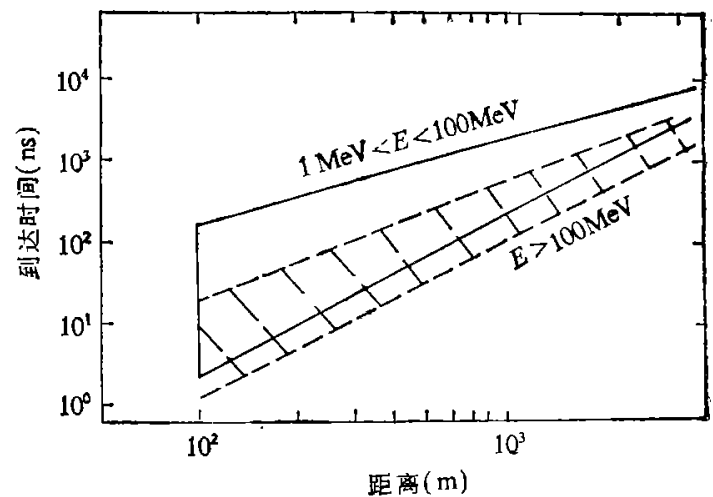

图 6 空气簇射粒子扁盘上能量的分布

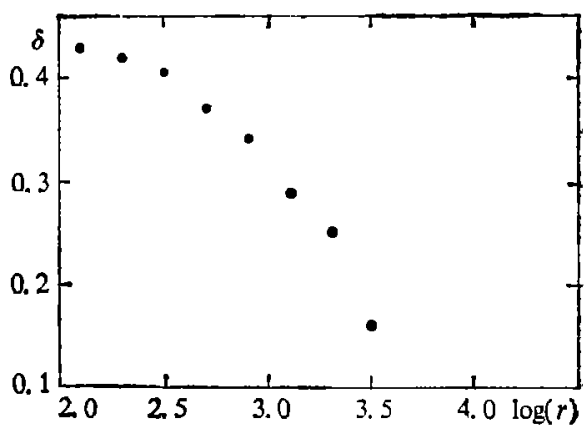

图 8 对数坐标下 $\mathrm{G}_{\mathrm{a}}$ uss 分布的标准 误差随距离的变化关系

在模拟中我们发现, $\langle\log (t)\rangle$ 几乎线性地随 $\log (r)$ 增加(图 7), 其中 $t$ 是到达时间, $r$ 是簇心距离. 如果近似地以 Gauss 分布来拟合次级粒子的到达时间分布 (在对数坐标下), Gauss 分布的均方差 $\delta$ 随距离的增加而减小(图 8). 通过很简单的拟合，我们得到到达时间. 的分布为

$$
\begin{gathered}
\langle\log (t)\rangle=-(2.45 \pm 0.05)+(1.85 \pm 0.02) \cdot \log r \\
-(0.033 \pm 0.004)(\log r)^{2}, \\
\delta=(0.015 \pm 0.018)+(0.424 \pm 0.007) \cdot \log r \\
-(0.107 \pm 0.001) \cdot(\log r)^{2} .
\end{gathered}
$$

在一定的近似程度上, 这两个公式可以用来构造 0-3000 $\mathrm{m}$ 之间簇射粒子的到达时间分 布。

\section{二、结 论}

从模拟中我们得出:

1. $\tau$ 和 $t r$ 与 $r$ 的关系陡于 $\sigma$ 与 $r$ 的关系;

2. 到达时间的分布几乎与能量和到达方向无关,也与原初成分无关;

3. 事例与事例之间的到达时间的分布的涨落很小，主要的涨落是由于有限的粒子数所引 
起的统计涨落;

4. 空气簇射扁盘主要是由电子和 $\boldsymbol{\mu}$ 子构成的,其最前峰部分是高能 $\boldsymbol{\mu}$ 子.

作者感谢东京大学明野观测站的成员，尤其是与永野教授的富有成效的讨论; 感谢参考文 献[1]的作者们对模拟程序的贡献; 感谢 Hazen 教授对戴宏跃的支持和鼓励。同时还感谢棚 桥先生和 Nakatska 的有益讨论. 模拟计算是在东京大学原子核研究所的 FACOM M 380 上 进行的,计算机室的成员给予了很大的帮助。

\section{参考文献}

[1] (a) Kasahara, K., Torii, S. \& Yuda, T., 16th ICRC, 13(1979), 70.

(b) Ding, L. K. et al., Proc. Ins. Symp. on Cosmic Rays ond Particle Physics, Institute For Cosmic Ray Research, Univ. of Tokyo, 1984, 142.

[ 2 ] Linsley, J., 19th ICRC, 3(1985), 434.

[ 3 ] Lonsley, J, J. Phys. G: Nucl. Phys., 12(1986), 51-57. 